\title{
大豆出苗期耐盐性鉴定方法建立及耐盐种质筛选
}

\author{
刘谢香 常奻镇关荣霞* 邱丽娟*
}

中国农业科学院作物科学研究所 / 国家农作物基因资源与遗传改良重大科学工程 / 农业部种质资源利用重点实验室, 北京 100081

摘 要: 土壤盐渍化是影响农业生产的重要问题, 筛选耐盐大豆资源对于大豆主产区盐渍化土壤的利用具有重要意

义。以中黄 35、中黄 39、Williams 82、铁丰 8 号、Peking 和 NY27-38 为供试材料, 以蛭石为培养基质, 设 $0 、 100$ 和 $150 \mathrm{mmol} \mathrm{L} \mathrm{L}^{-1} \mathrm{NaCl} 3$ 个处理, 进行出苗期耐盐性鉴定, 分析与生长相关的 6 个指标, 旨在明确大豆出苗期耐盐性鉴 定指标和评价方法。结果表明, $150 \mathrm{mmol} \mathrm{L} \mathrm{m}^{-1} \mathrm{NaCl}$ 处理显著降低大豆的成苗率、株高、地上部鲜重、根鲜重、地上 部干重和根干重, 并且不同材料间差异显著。基于幼苗生长发育状况的耐盐指数方法与耐盐系数方法对 6 份种质耐 盐性评价结果显著相关。耐盐指数法对植株无损坏、可省略种植对照, 节约人力和物力, 提高种质鉴定的效率。因此, 以 $150 \mathrm{mmol} \mathrm{L}^{-1} \mathrm{NaCl}$ 作为出苗期耐盐鉴定浓度, 以耐盐指数作为大豆出苗期耐盐鉴定评价指标, 鉴定 27 份大豆资源, 获得出苗期高度耐盐大豆(1 级) 3 份、耐盐大豆(2 级) 7 份, 其中 4 份苗期也高度耐盐(1 级), 分别为运豆 101、郑 1311、 皖宿 1015 和铁丰 8 号。本研究建立了一种以蛭石为基质, 利用 $150 \mathrm{mmol} \mathrm{L}^{-1} \mathrm{NaCl}$ 处理, 以耐盐指数作为评价指标的 大豆出苗期耐盐性鉴定评价的简便方法, 并筛选出 4 份出苗期和苗期均耐盐的大豆, 对耐盐大豆种质资源的高效鉴 定和耐盐大豆新品种培育具有重要意义。

关键词: 大豆; 出苗期; 耐盐性; 鉴定方法

\section{Establishment of screening method for salt tolerant soybean at emergence stage and screening of tolerant germplasm}

LIU Xie-Xiang, CHANG Ru-Zhen, GUAN Rong-Xia ${ }^{*}$, and QIU Li-Juan*

Institute of Crop Sciences, Chinese Academy of Agricultural Sciences / National Key Facility for Crop Gene Resources and Genetic Improvement (NFCRI) / Key Laboratory of Germplasm Utilization, Ministry of Agriculture, Beijing 100081, China

\begin{abstract}
Salinity is an important factor affecting crop production. Screening salt tolerant soybean germplasm is of great significance for the utilization of salinized soil in major soybean production regions. In order to select salt tolerant soybean, a screening method was developed by using six soybean accessions, including Zhonghuang 35, Zhonghuang 39, Williams 82, Tiefeng 8, Peking, and NY27-38. Seeds were grown in vermiculite and treated with 0,100 , and $150 \mathrm{mmol} \mathrm{L}^{-1} \mathrm{NaCl}$ solution. Seedling rate (SR), plant height (H), fresh weight of shoot and root (FWS and FWR), dry weight of shoot and root (DWS and DWR) were decreased significantly under $150 \mathrm{mmol} \mathrm{L}{ }^{-1} \mathrm{NaCl}$ treatment, with significant difference among varieties. Therefore, $150 \mathrm{mmol} \mathrm{L}$ $\mathrm{NaCl}$ was suitable to identify salt tolerant soybean at emergence stage. The salt tolerance index (SI) based on the growth and development of seedlings and the salt tolerance coefficient (ST) were significantly correlated with the salt tolerance. The method using salt tolerance index is non-destructive and does not require planting control, which could save time and labor in salt tolerant germplasm identification. Twenty-seven soybean resources were screened, in which three were highly tolerant (grade 1) and seven tolerant (grade 2) at emergence stage. Among them, Yundou 101, Zheng 1311, Wansu 1015, and Tiefeng 8 also showed salt tolerance (grade 1) at seedling stage. In summary, an effective method for screening salt tolerant soybean at emergence stage was developed, with vermiculite as the substrate, $150 \mathrm{mmol} \mathrm{L}^{-1} \mathrm{NaCl}$ as suitable treatment solution, and salt tolerance index as the indicator. Four soybean accessions were found to be salt tolerant at both emergence and seedling stages. This screening method will be useful for identification of salt tolerant soybean germplasm.
\end{abstract}


Keywords: soybean; emergence stage; salt tolerance; screening method

土壤盐渍化影响世界上 $20 \%$ 的可用耕地, 是导 致作物减产的重要因素之一 ${ }^{[1-3]}$ 。据全国第二次土壤 普查, 中国盐渍土总面积约 3600 万公顷, 占可利用 土地面积的 $4.88 \%{ }^{[4]}$ 。通过对不同作物盐度临界值 (不导致产量降低的最大土壤盐度)的研究发现, 大 豆属于中度耐盐作物，盐胁迫可抑制大豆的萌发、 生长发育和根瘤形成 ${ }^{[5-7]}$ 。当土壤盐度超过 $5 \mathrm{dS} \mathrm{m}^{-1}$ 时, 大豆的产量开始降低; 当土壤盐度为 $15 \mathrm{dS} \mathrm{m}^{-1}$ 时, 大豆产量显著降低甚至绝收 ${ }^{[8-9]}$ 。耐盐大豆品种 的培育是有效利用盐渍化土壤、促进大豆可持续发 展的重要途径 ${ }^{[2]}$ 。

大豆萌发期、出苗期、营养生长期和生殖生长 期等不同生育阶段的耐盐性没有明显相关性, 说明 不同时期可能存在不同的耐盐机制 ${ }^{[5,9-11]}$ 。大豆苗期 耐盐性的研究较为深入, 利用分子标记技术已经 检测到分布在 N、D2、G 等连锁群上的多个苗期 耐盐性相关的数量性状位点 (Quantitative Trait Locus, QTL), 特别是位于 $\mathrm{N}$ 连锁群的主效 QTL, 是在野生、栽培大豆不同耐盐资源中保守的重要 位点 ${ }^{[1-2,8,10,12-19]}$ 。本实验室前期在耐盐大豆铁丰 8 号 中图位克隆了位于 $\mathrm{N}$ 连锁群的苗期耐盐基因 $G m S A L T 3^{[17]}$, 在此基础上利用分子标记构建了分别 携带 GmSALT3 和 Gmsalt3 等位基因的近等基因系, 研究发现 GmSALT3 控制大豆苗期耐盐性, 但与大豆 出苗期的耐盐性无关, 进一步证明出苗期耐盐性可 能存在不同的遗传调控机制 ${ }^{[20]}$ 。有研究发现, 作物萌 发期的耐盐性远高于出苗期的耐盐性, 因此出苗期 耐盐性比萌发期耐盐性更具有研究和利用价值 ${ }^{[21-24]}$ 。

不同研究者对大豆萌发期和出苗期耐盐性的鉴 定方法、鉴定指标和评价方法也存在一些差异。鉴 定方法主要有田间鉴定和室内鉴定 2 类; 鉴定指标 有形态指标如受害叶面积、株高和生物量, 生长发 育指标如发芽率和出苗率, 以及生理生化指标如丙 二醛(MDA)含量和超氧化物歧化酶(SOD)活性等; 评价方法有盐害指数和耐盐系数法等 ${ }^{[5-6,24-29]}$ 。

本研究分析 6 份大豆种质盐胁迫条件下植株成 苗率、株高、地上部鲜重、根鲜重、地上部干重和 根干重, 建立了一种简便高效的大豆出苗期耐盐性 鉴定评价方法，并利用该方法进行大豆耐盐资源的 篮选, 为大豆耐盐优异资源发掘和新品种培育提供 了技术支撑。

\section{1 材料与方法}

\section{1 试验材料}

共 27 份种质, 其中用于方法建立的中黄 35、中 黄 39、Williams 82、铁丰 8 号、Peking 和 NY27-38 来自国家大豆种质资源库, 其余 21 份大豆新品种(系) 来自 2018 年国家黄淮海北片品种区域试验(表 3)。

\section{2 耐盐性鉴定}

1.2.1 出苗期耐盐鉴定与性状调查 耐盐性鉴定 于 2018 年 5 月至 6 月在中国农业科学院作物科学研究 所网室遮雨棚下进行。挑选每份大豆材料 90 粒饱满的 种子, 种于装有蛭石的 $8 \mathrm{~cm} \times 8 \mathrm{~cm} \times 8 \mathrm{~cm}$ 的小花盆中, 每盆 10 粒, 播种深度为 $2 \mathrm{~cm}$, 每 24 个小花盆置于一 个大蓝盒 $(46 \mathrm{~cm} \times 32 \mathrm{~cm} \times 10 \mathrm{~cm}$ )内, 用 $\mathrm{RO}$ 水(对照) 或盐溶液 $\left(100 \mathrm{mmol} \mathrm{L}^{-1} 、 150 \mathrm{mmol} \mathrm{L}^{-1} \mathrm{NaCl}\right)$ 处理, 3 次 重复。对照每个蓝盒中浇 $5 \mathrm{~L}$ 水, 盐处理每个蓝盒中分 别浇 $100 \mathrm{mmol} \mathrm{L}^{-1} 、 150 \mathrm{mmol} \mathrm{L}^{-1} \mathrm{NaCl}$ 溶液 $5 \mathrm{~L}$, 使蛭 石达到最大持水量, 此后每 3 天浇 $2 \mathrm{~L}$ 水。从第一个大 豆出苗(子叶突出蛭石表面)开始, 每天调查出苗数, 第 15 天调查成苗数(子叶展开、具有叶片的植株)。采 用单株分类记载法调查处理条件下所有材料的成苗情 况(图 1 和表 1)。测量成苗植株的株高, 称量地上部鲜 重、根鲜重, 随后于 $70^{\circ} \mathrm{C}$ 烘箱中烘干 $(3 \mathrm{~d})$, 分别称量 地上部和根干重, 计算相对成苗率(ST_SR)、相对株高 $(\mathrm{ST}$ H) 、相对地上部鲜重(ST_FWS)、相对根鲜重 (ST_FWR)、相对地上部干重(ST_DWS)和相对根干重 (ST_DWR)。各指标的相对值, 即耐盐系数(salt tolerance coefficient, ST), 为 $\mathrm{NaCl}$ 处理指标值/对照指标 值。耐盐指数( salt tolerance index, SI) $=\sum$ (类别数值 $\times$ 该类别株数 $)$ 播种粒数 $\times 5$ (最高类别数值)。

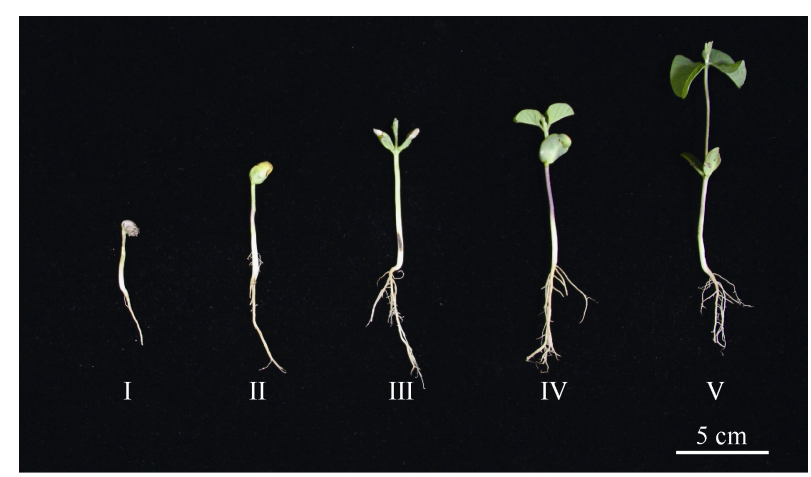

图 1 出苗期耐盐性单株分类记载法的标准

Fig. 1 Standard of individual classification for salt tolerance at germination stage 
表 1 盐胁迫后大豆出苗期和苗期的盐害症状

Table 1 Symptoms of soybean at emergence and seedling stages under salt stress

\begin{tabular}{|c|c|c|c|}
\hline \multicolumn{2}{|r|}{ 出苗期 Emergence stage } & \multicolumn{2}{|r|}{ 苗期 Seedling stage } \\
\hline $\begin{array}{c}\text { 类别 } \\
\text { Category }\end{array}$ & $\begin{array}{l}\text { 表型特征 } \\
\text { Symptom }\end{array}$ & $\begin{array}{l}\text { 级别 } \\
\text { Grade }\end{array}$ & $\begin{array}{l}\text { 表型特征 } \\
\text { Symptom }\end{array}$ \\
\hline I & $\begin{array}{l}\text { 植株凋亡, 子叶干枯(类别数值为 } 1 \text { ) } \\
\text { Plant dead, cotyledons were dry (category value is } 1 \text { ) }\end{array}$ & 1 & $\begin{array}{l}\text { 健康的绿叶, 没有观察到损伤 } \\
\text { Healthy green leaves, no damage observed }\end{array}$ \\
\hline II & $\begin{array}{l}\text { 植株生长受到严重抑制, 子叶未展开(类别数值为 } 2 \text { ) } \\
\text { Plant growth was severely inhibited, cotyledon was } \\
\text { not unfolded (category value is 2) }\end{array}$ & 2 & $\begin{array}{l}\text { 轻度坏死, 真叶轻微发黄 } \\
\text { Slight chlorosis, light yellowish color observed in true } \\
\text { leaves }\end{array}$ \\
\hline III & $\begin{array}{l}\text { 植株生长受到抑制, 具有生长点, 但真叶未展开(类 } \\
\text { 别数值为 3) } \\
\text { Plant growth was inhibited with shoot apical meristem, } \\
\text { but true leaves were not unfolded (category value is 3) }\end{array}$ & 3 & $\begin{array}{l}\text { 中度坏死, 三出复叶发黄 } \\
\text { Moderate chlorosis, chlorosis observed in trifoliate } \\
\text { leaves }\end{array}$ \\
\hline IV & $\begin{array}{l}\text { 植株生长基本正常, 真叶未完全展开(类别数值为 4) } \\
\text { Plant growth was basically normal, true leaves were } \\
\text { not fully expanded (category value is 4) }\end{array}$ & 4 & $\begin{array}{l}\text { 严重坏死，超过 } 75 \% \text { 的叶面发黄 } \\
\text { Severe chlorosis, more than } 75 \% \text { of the leaf area } \\
\text { showed chlorosis }\end{array}$ \\
\hline $\mathrm{V}$ & $\begin{array}{l}\text { 植株生长正常, 真叶完全展开(类别数值为 5) } \\
\text { Plant growth was normal, true leaves were fully ex- } \\
\text { panded (category value is 5) }\end{array}$ & 5 & $\begin{array}{l}\text { 凋亡, 植物完全枯萎 } \\
\text { Dead, plants were completely withered }\end{array}$ \\
\hline
\end{tabular}

1.2.2 苗期耐盐鉴定与性状调查 参照本实验室 前期建立的方法鉴定评价苗期耐盐性 ${ }^{[17]}$ 。每个品种 各 10 粒播于小花盆 $(8 \mathrm{~cm} \times 8 \mathrm{~cm} \times 8 \mathrm{~cm})$ 的蛭石中, 每 24 个小花盆置于一个大蓝盒内 $(46 \mathrm{~cm} \times 32 \mathrm{~cm} \times$ $10 \mathrm{~cm})$, 每个蓝盒浇 $5 \mathrm{~L} \mathrm{RO}$ 水。此后每 $3 \mathrm{~d}$ 浇 $2 \mathrm{~L}$ 水。待子叶完全展开后间苗, 每盆留 4 6 株。待真 叶完全展开后 $(10 \mathrm{~d})$, 每个蓝盒浇 $2 \mathrm{~L} 200 \mathrm{mmol} \mathrm{L}^{-1}$ $\mathrm{NaCl}$ ，于第 13 天和第 16 天再各浇 $2 \mathrm{~L} 200 \mathrm{mmol} \mathrm{L}^{-1}$ $\mathrm{NaCl}$, 设置 3 次重复。在最后一次盐处理 $5 \mathrm{~d}$ 后对叶 片坏死程度进行评估(表 1)。其中 1 级和 2 级为耐盐, $3 \sim 5$ 级为盐敏感。

\section{3 统计分析}

应用 Microsoft Excel 2010 处理数据, SAS9.4 软 件进行单因素方差分析, 最小显著极差法(Least Significant Differences, LSD)进行多重比较 $(P<0.05)$ 。

\section{2 结果与分析}

\subsection{6 份大豆种质出苗期耐盐性鉴定}

在 $100 \mathrm{mmol} \mathrm{L}{ }^{-1} \mathrm{NaCl}$ 处理下, 与对照相比, 6份 种质出苗正常且生长发育良好(图2-A, B), 成苗率、 地上部干重均与对照差异不显著(图2-D, I)。中黄35 (ZH35)的根鲜重和株高、NY27-38的根和地上部鲜 重较对照显著降低，中黄39 (ZH39)、Williams 82
(W82)、铁丰 8 号(TF8)和Peking (Pek)的根鲜重、株高 和地上部鲜重均与对照差异不显著(图2-E H)。

当 $\mathrm{NaCl}$ 浓度为 $150 \mathrm{mmol} \mathrm{L}^{-1}$ 时, 6 份种质出苗率 下降、株高降低、生长发育迟缓, 部分种子出苗后 子叶未展开, 不能正常 成苗(图1和图2-C)。除ZH39 和NY27-38的成苗率显著下降外, 其余材料的成 苗率与对照差异不显著; 6份种质的根鲜重、地上 部鲜重、株高、根干重和地上部干重均显著低于 对照(图2-D I)。不同种质间相对成苗率、相对地上 部鲜重、相对根鲜重、相对株高、相对地上部干重 和相对根干重差异显著(图3); 盐胁迫对不同的材料 的影响存在差异, W82、TF8、Pek和ZH39的成苗率、 地上部鲜重、根鲜重、株高、地上部干重和根重下 降程度较低, 耐盐性较强; 而ZH35和NY27-38的耐 盐性较差。因此, 确定 $150 \mathrm{mmol} \mathrm{L}{ }^{-1} \mathrm{NaCl}$ 为大豆出苗 期耐盐性鉴定适宜盐浓度。根据 6 个性状的耐盐系数 大小, 得出 6 个材料的耐盐性为W82 > Pek $>$ TF8 > ZH39 > ZH35 > NY27-38。

\section{2 大豆出苗期耐盐性鉴定的评价指标分析}

根据单株分类记载法计算耐盐指数, W82、Pek、 TF8、ZH39、ZH35 和 NY27-38 的耐盐指数分别为 $0.94 、 0.74 、 0.72 、 0.68 、 0.52$ 和 0.34 , 耐盐性以 $\mathrm{W} 82$ 最强, Pek、TF8 和 ZH39 次之, ZH35 和 NY27-38 最 

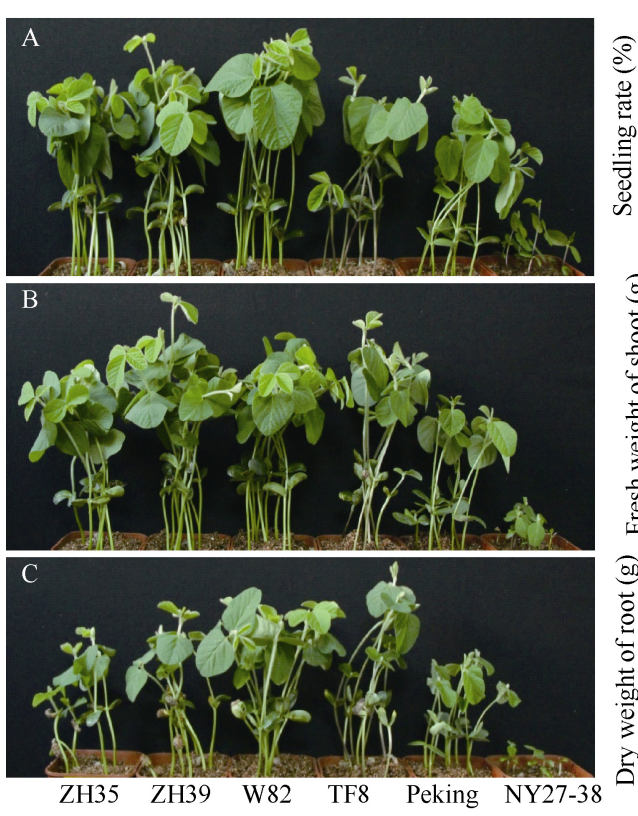
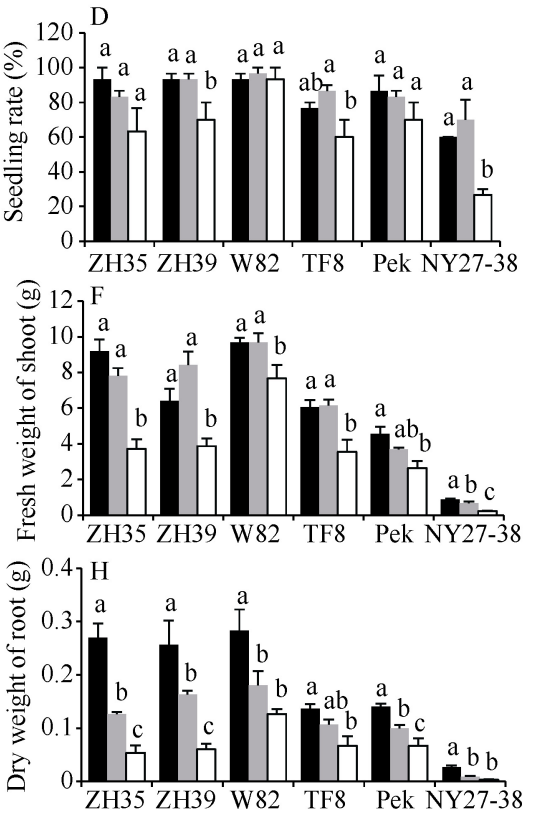
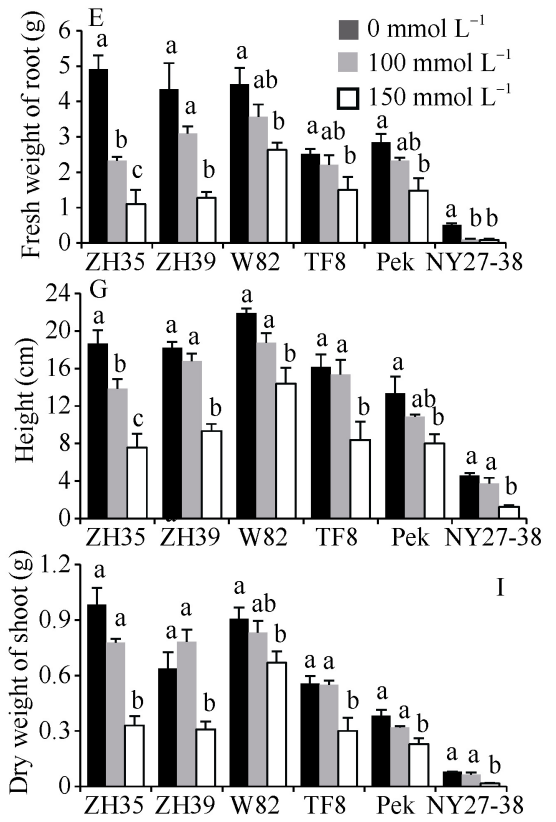

图 2 不同浓度 $\mathrm{NaCl}$ 处理 $\mathbf{1 5} \mathrm{d}$ 后 6 份材料的表型

Fig. 2 Phenotypes of six materials after treatment with $\mathrm{NaCl}$ solution for 15 days

$\mathrm{A} 、 \mathrm{~B} 、 \mathrm{C}$ 分别表示 $0 、 100$ 和 $150 \mathrm{mmol} \mathrm{L} \mathrm{L}^{-1} \mathrm{NaCl}$ 处理 $15 \mathrm{~d}$ 的表型特征, 比例尺为 $1 \mathrm{~cm}$ 。 $\mathrm{D}:$ 成苗率。 $\mathrm{E}$ : 根鲜重。 $\mathrm{F}$ : 地上部鲜重。 $\mathrm{G}$ : 株高。H: 根干重; I: 地上部干重。数据结果为 3 次生物学重复, 误差线为标准误 $(n=3)$; 标以不同小写字母的柱值在同一品种的 不同浓度处理间在 0.05 水平上差异显著。

$\mathrm{A}, \mathrm{B}$, and $\mathrm{C}$ refer to the phenotype after treatment with 0,100 , and $150 \mathrm{mmol} \mathrm{L}-1 \mathrm{NaCl}$ for 15 days, respectively. Bar $=1 \mathrm{~cm}$. D: seedling rate. E: fresh weight of root. F: fresh weight of shoot. G: shoot height. H: dry weight of root. I: dry weight of shoot. Data are mean \pm SE with three biological replicates $(n=3)$. Bars superscripted by different letters are significantly different at $P<0.05$ between different treatments of the same variety.

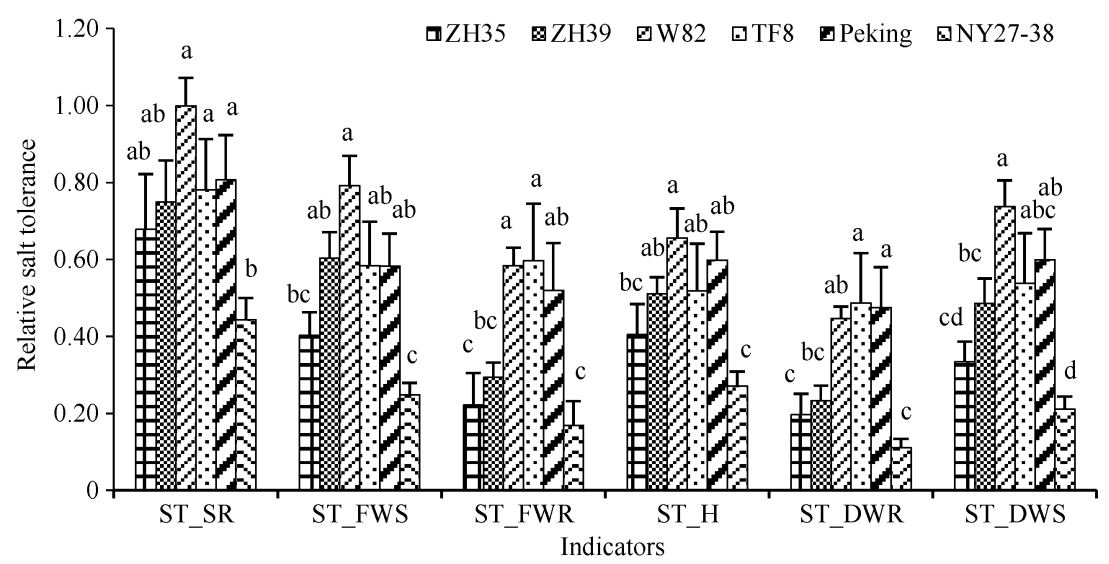

图 $3150 \mathrm{mmol} \mathrm{L}{ }^{-1} \mathrm{NaCl}$ 处理下 6 份材料的耐盐性

Fig. 3 Salt tolerance of six materials under $150 \mathrm{mmol} \mathrm{L}^{-1} \mathrm{NaCl}$ stress

ST_SR: 相对成苗率; ST_H: 相对株高; ST_FWR: 相对根鲜重; ST_FWS: 相对地上部鲜重; ST_DWR: 相对根干重; ST_DWS: 相对地 上部干重; 数据结果为 3 次生物学重复, 误差线为标准误 $(n=3)$; 标以不同小写字母的柱值在同一指标的不同品种间在 0.05 水平上差 异显著。

ST_SR: relative seedling rate; ST_H: relative height; ST_FWR: relative fresh weight of root; ST_FWS: relative fresh weight of shoot; ST_DWR: relative dry weight of root; ST_DWS: relative dry weight of shoot. Data are mean \pm SE with three biological replicates $(n=3)$. Bars superscripted by different letters are significantly different at $P<0.05$ between different varieties of the same indicator.

弱。相对成苗率、相对地上部鲜重、相对根鲜重、 相对株高、相对地上部干重、相对根干重和耐盐指 数7个指标的相关分析表明，除相对根干重与相对 成苗率、相对地上部鲜重相关不显著外, 其余性状
间均呈显著或极显著正相关; 相对地上部鲜重、相 对株高和相对地上部干重与相对成苗率高度相关; 相对鲜重与相对干重相关系数达 0.98 ; 耐盐指数与 6 个性状的耐盐系数均显著或极显著正相关(表2)。 
表 2 大豆出苗期不同耐盐评价指标间的相关系数

Table 2 Correlation coefficients between salt tolerance indexes at emergence stage in soybean

\begin{tabular}{|c|c|c|c|c|c|c|}
\hline $\begin{array}{l}\text { 指标 } \\
\text { Index }\end{array}$ & $\begin{array}{c}\text { 相对成苗率 } \\
\text { ST_SR }\end{array}$ & $\begin{array}{c}\text { 相对地上部鲜重 } \\
\text { ST_FWS }\end{array}$ & $\begin{array}{c}\text { 相对根鲜重 } \\
\text { ST_FWR }\end{array}$ & $\begin{array}{c}\text { 相对株高 } \\
\text { ST_H }\end{array}$ & $\begin{array}{c}\text { 相对根干重 } \\
\text { ST_DWR } \\
\end{array}$ & $\begin{array}{c}\text { 相对地上部干重 } \\
\text { ST_DWS } \\
\end{array}$ \\
\hline 相对地上部鲜重 ST_FWS & $0.97^{* * *}$ & & & & & \\
\hline 相对根鲜重 ST_FWR & $0.81^{*}$ & $0.81^{*}$ & & & & \\
\hline 相对株高 ST_H & $0.97^{* * *}$ & $0.96^{* *}$ & $0.85^{*}$ & & & \\
\hline 相对根干重 ST_DWR & 0.80 & 0.77 & $0.98^{* * *}$ & $0.85^{*}$ & & \\
\hline 相对地上部干重 ST_DWS & $0.97^{* * *}$ & $0.98^{* * *}$ & $0.89^{*}$ & $0.99^{* * *}$ & $0.87^{*}$ & \\
\hline 耐盐指数 SI & $0.88^{*}$ & $0.83^{*}$ & $0.91^{* *}$ & $0.87^{*}$ & $0.88^{*}$ & $0.90^{* *}$ \\
\hline
\end{tabular}

"表示显著 $(P<0.05),{ }^{* *}$ 表示极显著 $(P<0.01),{ }^{* * *}$ 表示极显著 $(P<0.001)$ 。

* represent significance level at $P<0.05,{ }^{* *}$ represent significance level at $P<0.01$, and ${ }^{* * *}$ represent significance level at $P<0.001$. ST_SR: relative seedling rate. ST_H: relative height. ST_FWR: relative fresh weight of root. ST_FWS: relative fresh weight of shoot. ST_DWR: relative dry weight of root. ST_DWS: relative dry weight of shoot.

耐盐系数法是经典的出苗期耐盐性评价方法, 通过盐处理与对照的比值反映盐胁迫使大豆成苗 数、生长量下降的程度。耐盐指数法同时考虑了盐 胁迫后大豆的出苗数量及幼苗生长发育状况; 在实 际操作方面, 省略了种植对照, 实现了耐盐性状无 损调查, 可极大节约人力和物力, 为大批量优异种 质的快速鉴定提供了可能。

\section{3 大豆耐盐种质篮选}

分别以Williams 82 和中黄 35 为耐盐和盐敏感对 照, 用 $150 \mathrm{mmol} \mathrm{L}^{-1} \mathrm{NaCl}$ 对包括上述6份在内的27份
大豆种质进行耐盐性鉴定(图4), $15 \mathrm{~d}$ 后根据单株分 类记载法计算耐盐指数, 可将 27 份种质的出苗期耐 盐性分为 5 级, 1 级为高度耐盐型 $(0.80 \leq \mathrm{SI}<1.00)$, 2 级 为耐盐型 $(0.60 \leq \mathrm{SI}<0.80), 3$ 级为中度耐盐型 $(0.40 \leq$ $\mathrm{SI}<0.60), 4$ 级为敏感型 $(0.20 \leq \mathrm{SI}<0.40)$, 5 级为高度敏 感型 $(0.00 \leq \mathrm{SI}<0.20)$, 其中 1 级 3 份, 2 级7 份(表3)。同 时, 用 $200 \mathrm{mmol} \mathrm{L}^{-1} \mathrm{NaCl}$ 对27份大豆品种(系)进行 苗期耐盐鉴定, 发现12份材料苗期高度耐盐(1级)。 其中, 运豆101、郑1311、浣宿 1015 和铁丰 8 号是出 苗期和苗期均耐盐的大豆种质(表3)。

\section{表 327 份大豆种质苗期和出苗期的耐盐等级}

Table 3 Salt tolerance of 27 accessions at seedling and emergence stages

\begin{tabular}{|c|c|c|c|c|c|}
\hline \multirow{2}{*}{$\begin{array}{c}\text { 品种 } \\
\text { Variety }\end{array}$} & \multicolumn{2}{|c|}{ 耐盐性 Salt tolerance } & \multirow{2}{*}{$\begin{array}{c}\text { 品种 } \\
\text { Variety }\end{array}$} & \multicolumn{2}{|c|}{ 耐盐性 Salt tolerance } \\
\hline & $\begin{array}{c}\text { 出苗期 } \\
\text { Emergence stage }\end{array}$ & $\begin{array}{c}\text { 苗期 } \\
\text { Seedling stage }\end{array}$ & & $\begin{array}{c}\text { 出苗期 } \\
\text { Emergence stage }\end{array}$ & $\begin{array}{c}\text { 苗期 } \\
\text { Seedling stage }\end{array}$ \\
\hline 中黄 74 Zhonghuang 74 & 3 & 5 & 运豆 101 Yundou 101 & 2 & 1 \\
\hline 冀 1507 Ji 1507 & 3 & 1 & 郑 1311 Zheng 1311 & 2 & 1 \\
\hline 冀豆 29 Jidou 29 & 3 & 5 & 冀 1503 Ji 1503 & 4 & 1 \\
\hline 冀豆 23 Jidou 23 & 4 & 1 & 皖宿 1015 Wansu 1015 & 2 & 1 \\
\hline 安豆 1498 Andou 1498 & 1 & 5 & 齐黄 39 Qihuang 39 & 1 & 4 \\
\hline 石豆 17 Shidou 17 & 4 & 1 & 中黄 207 Zhonghuang 207 & 3 & 3 \\
\hline 科豆 $13 \mathrm{Kedou} 13$ & 4 & 4 & 邯豆 11 Handou 11 & 3 & 5 \\
\hline 中黄 206 Zhonghuang 206 & 4 & 3 & Williams 82 & 1 & 4 \\
\hline 中黄 605 Zhonghuang 605 & 2 & 5 & 中黄 35 Zhonghuang 35 & 3 & 5 \\
\hline 中黄 80 Zhonghuang 80 & 3 & 3 & 中黄 39 Zhonghuang 39 & 2 & 5 \\
\hline 中黄 203 Zhonghuang 203 & 3 & 1 & 铁丰 8 号 Tiefeng 8 & 2 & 1 \\
\hline 中黄 204 Zhonghuang 204 & 3 & 1 & Peking & 2 & 5 \\
\hline 中黄 70 Zhonghuang 70 & 3 & 5 & NY27-38 & 4 & 1 \\
\hline 圣豆 10 号 Shengdou 10 & 3 & 1 & & & \\
\hline
\end{tabular}




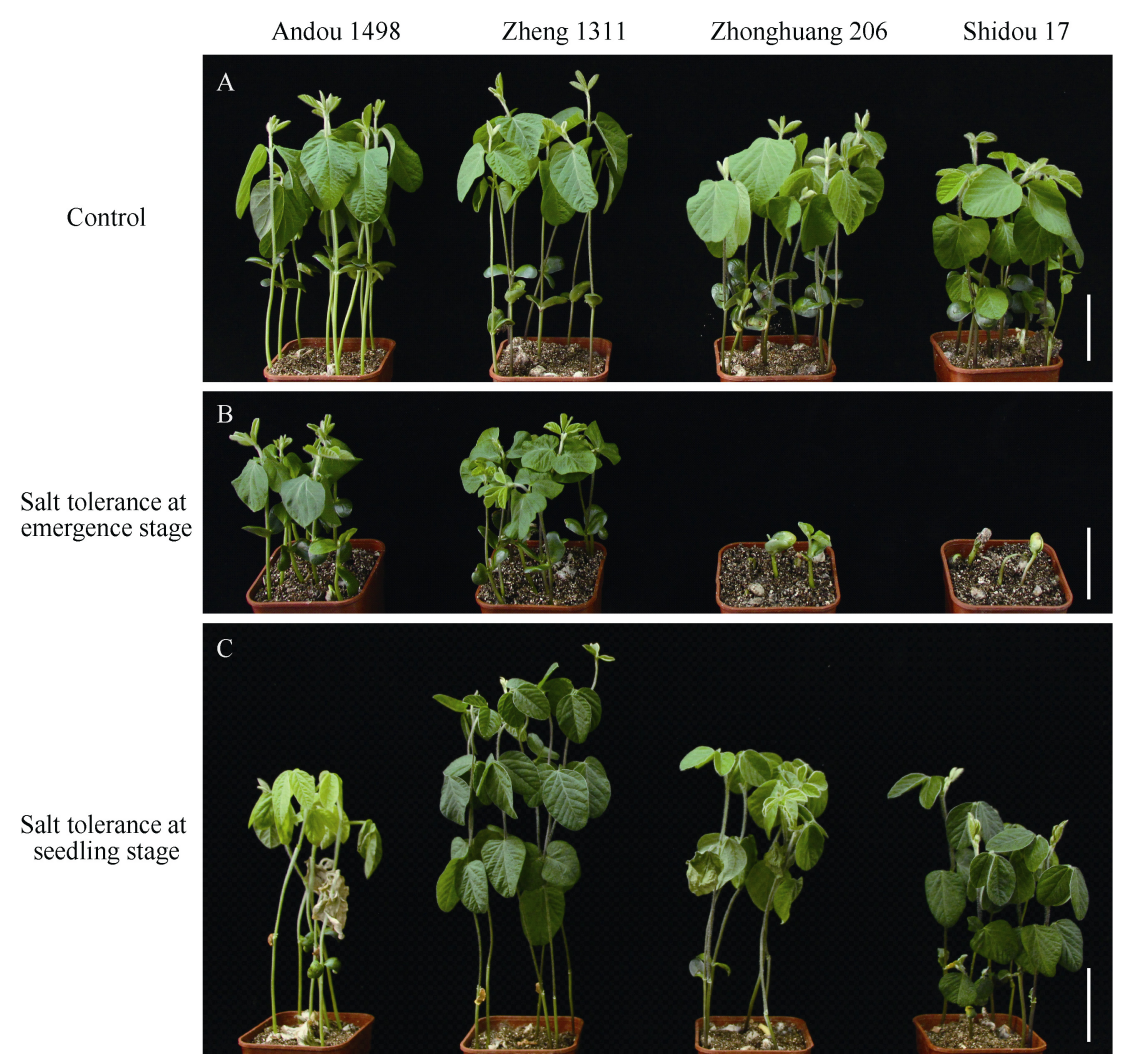

图 44 份大豆种质苗期和出苗期耐盐性

Fig. 4 Salt tolerance in both seedling and emergence stages for four accessions

A：对照 $\left(0 \mathrm{mmol} \mathrm{L}^{-1} \mathrm{NaCl}\right.$ 处理 $\left.15 \mathrm{~d}\right) 。 \mathrm{~B}$ ：出苗期耐盐性(播种时 $150 \mathrm{mmol} \mathrm{L}^{-1} \mathrm{NaCl}$ 处理 $15 \mathrm{~d}$ )。C：苗期耐盐性(真叶展开时 $200 \mathrm{mmol} \mathrm{L}^{-1}$ $\mathrm{NaCl}$ 处理 $15 \mathrm{~d})$ 。比例尺 $=5 \mathrm{~cm}$ 。

A: control $\left(0 \mathrm{mmol} \mathrm{L} \mathrm{L}^{-1} \mathrm{NaCl}\right.$ treatment for 15 days $)$. B: salt tolerance at emergence stage $\left(150 \mathrm{mmol} \mathrm{L}^{-1} \mathrm{NaCl}\right.$ treatment for 15 days after sowing). C: salt tolerance at seedling stage $\left(200 \mathrm{mmol} \mathrm{L}^{-1} \mathrm{NaCl}\right.$ treatment for 15 days when the unifoliate leaves of plants were fully expanded). Bars $=5 \mathrm{~cm}$.

\section{3 讨论}

\section{1 大豆出苗期耐盐性鉴定方法}

在盐碱地上,“拿住苗”是大豆生产的关键问题, 因此，出苗期的耐盐性十分重要 ${ }^{[24]}$ 。大豆为中度耐 盐作物，耐盐性不同的大豆种质资源盐害症状存在 明显差异 ${ }^{[9]}$ 。国内外学者采用了多种不同的方法评 价大豆出苗期的耐盐性。邵桂花等 ${ }^{[11]}$ 通过抽提地下 咸水和淡水配制成一定浓度的咸水对2000多个大豆 品种进行处理, 根据盐害指数法筛选出出苗期耐盐 品种242个。田间鉴定方便快捷, 适用于大批量品种 的鉴定。但田间漫灌会导致盐分分布不均匀，如地 头土壤盐浓度仅为 $3 \mathrm{dS} \mathrm{m}^{-1}$, 而地尾的土壤盐浓度 则高达 $11 \mathrm{dS} \mathrm{m}^{-1}$ [6]。田间鉴定法还易受地力、光照、 气温、降水和风力的影响, 可重复性较差, 因此, 需 要严格控制鉴定条件 ${ }^{[24,30]}$ 。罗庆云等 ${ }^{[25]}$ 利用营养液 水培法鉴定大豆出苗期的耐盐性, 发现 $\mathrm{NaCl}$ 胁迫降 低种子出苗速率, 影响幼苗正常生长, 但需多次
更换营养液，费时费力。张海波等 ${ }^{[26]}$ 鉴定发现耐盐 性较强的大豆品种较对照 MDA 含量降低, SOD 活性 升高，但生理生化指标易受胁迫方式、胁迫时间、 测定部位、测定方式和所用仪器设备等因素影响。 为模拟大田环境, 本研究以蛭石为基质, 在遮雨棚 下利用自然光温条件进行大豆种质资源的耐盐性鉴 定。与田间鉴定法相比，该方法不受地力、降水等 影响, 可重复性高。同时, 蛭石吸水迅速, 有利于保 持土壤盐分的相对稳定，且无需更换溶液或配制混 合基质，具有快速、准确、经济等特点。

邵桂花等 ${ }^{[11]}$ 在评价大豆出苗期耐盐性时采用的 盐水浓度为 $10 \sim 15 \mathrm{mS} \mathrm{cm}^{-1}$ 。Zhang 等 ${ }^{[27]}$ 鉴定所利用 浓度为 $100 \mathrm{mmol} \mathrm{L}^{-1} \mathrm{NaCl}_{\text {。罗庆云等 }}{ }^{[25]}$ 研究发现, 在 $50 \mathrm{mmol} \mathrm{L}^{-1}$ 和 $100 \mathrm{mmol} \mathrm{L}^{-1} \mathrm{NaCl}$ 营养液胁迫下, 所有参试大豆均能形成部分正常植株, 而在 $150 \mathrm{mmol} \mathrm{L}^{-1} \mathrm{NaCl}$ 处理下则不能正常发育成苗。邵 桂花等 ${ }^{[24]}$ 认为 $\mathrm{NaCl}$ 溶液浓度可根据试验目的进行 调整, 若为评价耐盐性, 则鉴定浓度可低些, 若篮 
选高度耐盐种质, 则可适当提高浓度。本研究中用 $100 \mathrm{mmol} \mathrm{L}^{-1} \mathrm{NaCl}$ 处理, 所有种质生长发育良好, 相对盐敏感的种质 ZH35 和 NY27-38 的成苗率、地 上部鲜重、株高和地上部干重与对照差异不显著; 而 $150 \mathrm{mmol} \mathrm{L}^{-1} \mathrm{NaCl}$ 处理下, 所有种质的性状均与 对照差异显著, 且不同品种受盐胁迫影响的程度差 异明显。因此, 以 $150 \mathrm{mmol} \mathrm{L}^{-1} \mathrm{NaCl}$ 作为大豆出苗 期耐盐性评价鉴定浓度。

邵桂花等 ${ }^{[24]}$ 通过受害叶面积为分类标准计算 盐害指数评价大豆出苗期的耐盐性, 但盐害症状 的分辨会因研究人员的经验不同而产生差异 ${ }^{[25]}$ 。 本研究观察到子叶出土后, 盐胁迫对其发育影响 较大, 有的子叶无法展开最终干枯凋亡, 也有的 能正常生长。因此, 根据幼苗发育情况, 将其分为 5 级(图 1), 在评价时将出苗数与生长发育状况级 别相结合, 计算耐盐指数, 可准确地评价大豆种 质出苗期的耐盐性。此外, 单株分类记载法只统计 处理条件下品种出苗情况的差异性, 不用种植对 照也不损伤植株, 从而节约大量的劳动成本, 适 用于大批量种质资源的高效鉴定, 也为其他作物 耐盐性鉴定提供了借鉴。

\section{2 大豆不同发育阶段的耐盐性研究}

作物在不同生长发育阶段对盐分的敏感性存 在差异 ${ }^{[31]}$ 。例如, 水稻的芽期耐盐性高于生殖生长 期 ${ }^{[22]}$ 。番茄萌发期与幼苗生长发育阶段的耐盐性不 同 ${ }^{[32]}$ 。同一大豆种质不同发育阶段耐盐性也存在差 异, 如大豆栽培品种 Lee、Coiquitt 和 Clark 36 受盐 胁迫后发芽率降低的程度相似，而 Lee 苗期耐盐性 高于 Coiquitt 和 Clark $36^{[15]}$ 。研究发现, 大豆萌发期 的耐盐性强于出苗期和苗期, 以后随生育期的增进 耐盐性增强 ${ }^{[1,23]}$ 。当 $\mathrm{NaCl}$ 浓度为 $220 \mathrm{mmol} \mathrm{L}^{-1}$ 时, 种子的萌发率几乎不受影响, 而此浓度下幼苗生长 率较对照下降 95\%; $\mathrm{NaCl}$ 提高到 $330 \mathrm{mmol} \mathrm{L}^{-1}$ 时, 幼苗的生长完全被抑制, 而种子的萌发率仍可达 $81 \%$; 当胚轴中的 $\mathrm{Na}^{+}$含量为 $9.3 \mathrm{mg} \mathrm{g}^{-1} \mathrm{FW}$ 时, 部分 种子仍能萌发 $(40 \%)$, 而幼苗的生长则在 $\mathrm{Na}^{+}$含量为 $6.1 \mathrm{mg} \mathrm{g}^{-1} \mathrm{FW}$ 时受到完全抑制 ${ }^{[23]}$ 。这些结果表明, 与后期生长相比, 大豆芽期能耐受更高的盐浓度, 种子萌发并不意味着能够正常生长成幼苗。此外, 大田播种通常会在雨后进行，此时田间土壤的盐度 会较低。因此, 出苗期的耐盐性研究对于大豆生产 更具有指导意义 ${ }^{[21]}$ 。

目前, 已克隆了大豆苗期耐盐关键基因 GmSALT3/

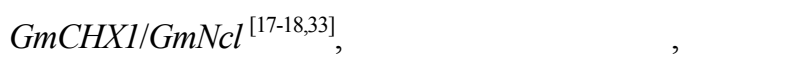
因 GmSALT3 主要通过增加粒重, 使耐盐大豆增产 $30 \% \sim 50 \%{ }^{[20]}$ 。而出苗期耐盐基因挖掘的研究较少。 Zhang 等 ${ }^{[27]}$ 利用 257 个大豆品种与 135 个 SSR 标记 进行出苗期耐盐性的关联分析，检测到 83 个与环境 互作的微效 QTL (解释的表型贡献率：1\% 5\%)。因 此, 本研究建立的大豆出苗期耐盐鉴定方法对于篮 选耐盐种质资源、发掘耐盐基因、培育耐盐品种具 有十分重要的意义。

\section{4 结论}

建立了一种以蛭石为基质, 利用 $150 \mathrm{mmol} \mathrm{L}^{-1}$ $\mathrm{NaCl}$ 处理 $15 \mathrm{~d}$ 的简便快速鉴定方法, 不需要种植对 照, 只需统计处理条件下出苗数及每株苗的耐盐等 级, 计算耐盐指数作为评价大豆出苗期耐盐性指 标。利用该方法篮选到出苗期耐盐大豆种质 10 份, 为耐盐大豆品种的培育提供基础材料和鉴定方法。

\section{References}

[1] Hamwieh A, Xu D. Conserved salt tolerance quantitative trait loci (QTL) in wild and cultivated soybean. Breed Sci, 2008, 58: 355-359.

[2] Hamwieh A, Tuyen D D, Cong H, Benitez E R, Takahashi R, Xu D H. Identification and validation of a major QTL for salt tolerance in soybean. Euphytica, 2011, 179: 451-459.

[3] Wang Z, Wang J, Bao Y, Wu Y, Zhang H. Quantitative trait loci controlling rice seed germination under salt stress. Euphytica, 2011, 178: 297-307.

[4] 王佳丽, 黄贤金, 钟太洋, 陈志刚. 盐碱地可持续利用研究综 述. 地理学报, 2011, 66: 673-684.

Wang J L, Huang X J, Zhong T Y, Chen Z G. Review on sustainable utilization of salt-affected land. Acta Geogr Sin, 2011, 66: 673-684 (in Chinese with English abstract).

[5] Abel G H, Mackenzie A J. Salt tolerance of soybean varieties (Glycine $\max$ L. Merrill) during germination and later growth. Crop Sci, 1964, 4: 14-28.

[6] Wang D, Shannon M C. Emergence and seedling growth of soybean cultivars and maturity groups under salinity. Plant Soil, 1999, 214: 117-124.

[7] Singleton P W, Bohlool B B. Effect of salinity on nodule formation by soybean. Plant Physiol, 1984, 74: 72-76.

[8] Chen H, Cui S, Fu S, Gai J, Yu D. Identification of quantitative trait loci associated with salt tolerance during seedling growth in soybean (Glycine $\max$ L.). Aust J Agric Res, 2008, 59: 1086-1091.

[9] Phang T H, Lam H M. Salt tolerance in soybean. J Integr Plant Biol, 2008, 50: 1196-1212.

[10] Pathan M S, Lee J D, Shannon J G, Nguyen H T. Recent advances in breeding for drought and salt stress tolerance in soybean. In: Jenks M A, Hasegawa P M, Jain S M, eds. Advances in Molecular-breeding Toward Drought and Salt Tolerant Crops. USA: 
Springer, 2007. pp 739-773.

[11] 邵桂花, 宋景芝, 刘惠令. 大豆种质资源耐盐性鉴定初报. 中 国农业科学, 1986, 19(6): 30-35.

Shao G H, Song J Z, Liu H L. Preliminary studies on the evaluation of salt tolerance in soybean varieties. Sci Agric Sin, 1986, 19(6): 30-35 (in Chinese with English abstract).

[12] 郭蓓, 邱丽娟, 邵桂花, 许占友. 大豆耐盐性种质的分子标记 辅助鉴定及其利用研究. 大豆科学, 2002, 21: 56-61.

Guo B, Qiu L J, Shao G H, Xu Z Y. Markers-assisted identification of the salt tolerant accessions in soybean. Soybean Sci, 2002, 21: 56-61 (in Chinese with English abstract).

[13] Ha B K, Vuong T D, Velusamy V, Nguyen H T, Shannon J G, Lee J D. Genetic mapping of quantitative trait loci conditioning salt tolerance in wild soybean (Glycine soja) PI 483463. Euphytica, 2013, 193: 79-88.

[14] Tuyen D D, Lal S K, Xu D H. Identification of a major QTL allele from wild soybean (Glycine soja Sieb. \& Zucc.) for increasing alkaline salt tolerance in soybean. Theor Appl Genet, 2010, 121: 229-236.

[15] Essa T A. Effect of salinity stress on growth and nutrient composition of three soybean (Glycine $\max$ L. Merrill) cultivars. $J$ Agron Crop Sci, 2002, 188: 86-93.

[16] Tuyen D D, Zhang H M, Xu D H. Validation and high-resolution mapping of a major quantitative trait locus for alkaline salt tolerance in soybean using residual heterozygous line. Mol Breed, 2013, 31: 79-86.

[17] Guan R X, Qu Y, Guo Y, Yu L L, Liu Y, Jiang J H, Chen J G, Ren Y L, Liu G Y, Tian L, Jin L G, Liu Z X, Hong H L, Chang R Z, Gilliham M, Qiu L J. Salinity tolerance in soybean is modulated by natural variation in GmSALT3. Plant J, 2015, 80: 937-950.

[18] Qi X, Li M W, Xie M, Liu X, Ni M, Shao G, Song C, Yim K Y, Tao Y, Wong F L, Isobe S, Wong C F, Wong K S, Xu C, Li C, Wang Y, Guan R, Sun F, Fan G, Xiao Z, Zhou F, Phang T H, Liu X, Tong S W, Chan T F, Yiu S M, Tabata S, Wang J, Xu X, Lam H M. Identification of a novel salt tolerance gene in wild soybean by whole-genome sequencing. Nat Commun, 2014, 5: 4340.

[19] Do T D, Vuong T D, Dunn D, Smothers S, Patil G, Yungbluth D C, Chen P, Scaboo A, Xu D, Carter T E. Mapping and confirmation of loci for salt tolerance in a novel soybean germplasm, Fiskeby III. Theor Appl Genet, 2018, 131: 513-524.

[20] Liu Y, Yu L L, Qu Y, Chen J J, Liu X X, Hong H L, Liu Z X, Chang R Z, Gilliham M, Qiu L J, Guan R X. GmSALT3, which confers improved soybean salt tolerance in the field, increases leaf $\mathrm{Cl}^{-}$exclusion prior to $\mathrm{Na}^{+}$exclusion but does not improve early vigor under salinity. Front Plant Sci, 2016, 7: 1485.

[21] Munns R, James R A. Screening methods for salinity tolerance: a case study with tetraploid wheat. Plant Soil, 2003, 253: 201-218.

[22] Khatun S, Flowers T J. Effects of salinity on seed set in rice.
Plant Cell Environ, 1995, 18: 61-67.

[23] Hosseini M, Powell A, Bingham I. Comparison of the seed germination and early seedling growth of soybean in saline conditions. Seed Sci Res, 2002, 12: 165-172.

[24] 邵桂花. 大豆种质资源耐盐性田间鉴定方法. 作物杂志, 1986, (3): $36-37$.

Shao G H. Field identification method for salt tolerance of soybean germplasm resources. Crops, 1986, (3): 36-37 (in Chinese).

[25] 罗庆云. 野生大豆和栽培大豆耐盐机理及遗传研究. 南京农 业大学博士学位论文, 江苏南京, 2003 .

Luo Q Y. Study on Mechanism and Inheritance of Salt Tolerance in Wild Soybean (Glycine soja) and Cultivated Soybean (G. max). $\mathrm{PhD}$ Dissertation of Nanjing Agriculture University, Nanjing, China, 2003 (in Chinese with English abstract).

[26] 张海波, 崔继哲, 曹甜甜, 张佳䑣, 刘千千, 刘欢. 大豆出苗 期和苗期对盐胁迫的响应及耐盐指标评价. 生态学报, 2011, 31: 2805-2812.

Zhang H B, Cui J Z, Cao T T, Zhang J T, Liu Q Q, Liu H. Response to salt stresses and assessment of salt tolerability of soybean varieties in emergence and seedling stages. Acta Ecol Sin, 2011, 31: 2805-2812 (in Chinese with English abstract).

[27] Zhang W J, Niu Y, Bu S H, Li M, Feng J Y, Zhang J, Yang S X, Odinga M M, Wei S P, Liu X F, Zhang Y M. Epistatic association mapping for alkaline and salinity tolerance traits in the soybean germination stage. PLoS One, 2014, 9: e84750.

[28] Kan G, Zhang W, Yang W, Ma D, Zhang D, Hao D, Hu Z, Yu D. Association mapping of soybean seed germination under salt stress. Mol Genet Genomics, 2015, 290: 2147-2162.

[29] 姬丹丹, 刘畅, 曹其聪, 向凤宁. 不同大豆品种芽期和苗期耐 盐性比较研究. 山东轻工业学院学报(自然科学版), 2011, 25(2): 4-7.

Ji D D, Liu C, Cao Q C, Xiang F N. Comparison of the salt tolerance at sprout and seedling in different soybean. $J$ Shandong Polytechnic Univ (Nat Sci Edn), 2011, 25(2): 4-7 (in Chinese with English abstract).

[30] 郭蓓, 邵桂花. 大豆耐盐基因的 PCR 标记. 中国农业科学, 2000, 33(1): 10-16.

Guo B, Shao G H. Tagging salt tolerant gene using PCR marker in soybean. Sci Agric Sin, 2000, 33(1): 10-16 (in Chinese with English abstract).

[31] Flowers T J. Improving crop salt tolerance. J Exp Bot, 2004, 55: 307-319.

[32] Foolad M R, Lin G Y. Absence of genetic relationship between salt tolerance during seed germination and vegetative growth in tomato. Plant Breed, 1997, 116: 363-367.

[33] Do T D, Chen H, Hien V T T, Hamwieh A, Yamada T, Sato T, Yan Y, Cong H, Shono M, Suenaga K. $N c l$ synchronously regulates $\mathrm{Na}^{+}, \mathrm{K}^{+}$, and $\mathrm{Cl}^{-}$in soybean and greatly increases the grain yield in saline field conditions. Sci Rep, 2016, 6: 19147. 